\title{
EMOTIONAL DISTRESS AS A RISK FACTOR FOR CORONARY HEART DISEASE IN CLINICALLY HEALTHY MEN WITH ALEXITHYMIA
}

\begin{abstract}
Anna Kodochigova ${ }^{\bowtie}$, Tatyana Bogdanova, Diana Psanukova, Vyacheslav Kirichuk, Mikhail Sinkeev, Elena Olenko (D), Melek Dzheyranova, Victoria Blinova, Maxim Zankin, Valeria Semenova
\end{abstract}

V. Razumovsky Saratov State Medical University, Saratov, Russia

\author{
kodochigovaa@yandex.ru
}

years), since male sex is an independent risk factor for the development and complicated course of coronary heart disease [3.6]; all of them were blood donors. To identify the psychological characteristics of the individual, we used: The Toronto Alexithymic Scale (TAS) [2], questionnaires of Ch.SpielbergerYu.Khanina for the assessment of reactive and personal anxiety [11] and S. Subbotina for the establishment of stress resistance levels [10]. The statistical analysis of the obtained data was carried out using the Statgraphics Centurion 19.1.2 program. The reliability of the statistical estimates used was at least $95 \%$.

\section{RESULTS}

As a result of testing according to the data of the persons we observed, it was found that more than half of the respondents had signs of alexithymia of varying severity (20.9\% were carriers of it, and $32.4 \%$ were in the risk zone of its development); the average indicators of this test in the general group were 64.1 points which corresponds to the risk zone.

Further psychological analysis of the healthy male individuals we observed was carried out taking into account the gradation according to TAS indicators: Subgroup I consisted of men with obvious clinical signs of alexithymia; subgroup II consisted of persons classified as at risk and subgroup III consisted of persons having indicators corresponding to the physiological norm.

It was found that the carriers of alexithymia had indicators of personal anxiety at moderate and high levels (there was no low level of anxiety as a disposition); and respondents without deviations from the norm of TAS indicators, along with medium and high levels of anxiety as a disposition, had low personal anxiety in $1 / 5$ of cases (Fig. 1, $\mathrm{p}<0,05$ ).

Men who are not resistant to the action of stress agents were found only in the subgroup of carriers of alexithymia, and the largest proportion of respondents with high stress resistance was detected in the subgroup of clinically healthy men without signs of alexithymia (Fig. 2, p $<0,05$ ). 


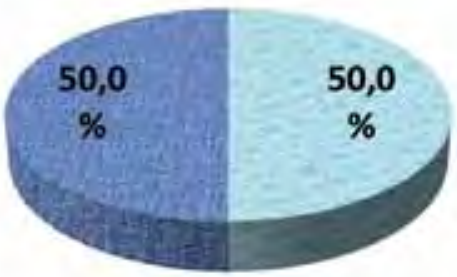

1 내 2 : 3

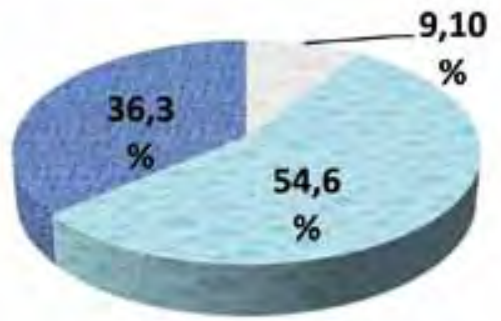

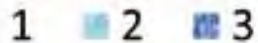

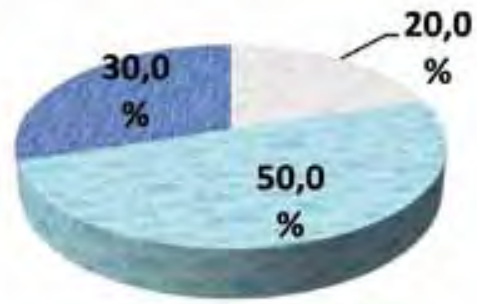

$1 \div 2$ : 3

$A$

$B$

C

Fig. 1. Distribution of indicators of personal anxiety in the individuals observed in the context of alexithymia

Note. Values of anxiety as a disposition: $A$ - for carriers of alexithymia; $B$ - for persons who are at "risk" of developing alexithymia; $C$ - in the absence of deviations from the norm of TAS indicators. Levels of personal anxiety: 1 - low; 2 - moderate; 3 - high

14,2

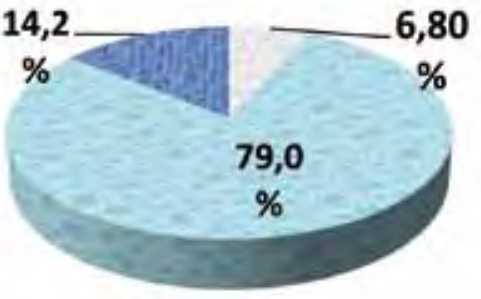

1 a 2 춭 3

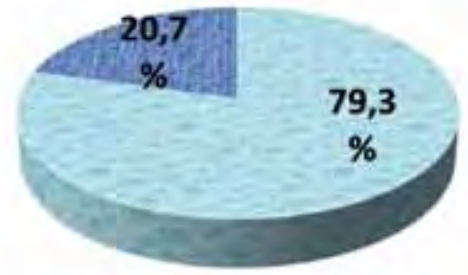

1 : 2 : 3

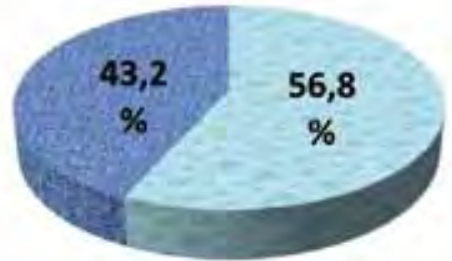

$1 \quad 2$ 르 3

A

B

Fig. 2. Distribution of indicators of resistance to the action of stress agents in the individuals observed in the context alexithymia.

Note. Values of resistance to the action of stress agents: A — for carriers of alexithymia; B — for persons who are at "risk" of developing alexithymia; C — in the absence of deviations from the norm of TAS indicators. Levels of resistance to the action of stress agents: 1 — low (unstable); 2 - medium; 3-high

\section{DISCUSSION OF THE RESULTS}

Alexithymia, as a psycho-emotional characteristic of a person and an independent risk factor for the development of neuroses and psychosomatic pathology, including cardiovascular (and, first of all, CHD), is increasingly found not only in various psychosomatic diseases, but also in clinically healthy individuals $[8,9]$. So, in our study, in clinically healthy men who are blood donors, the average test results for TAS allowed them to be attributed to the risk group for the development of alexithymia. During detailing the results of this test, the predominance of male individuals with a tendency to develop alexithymia and its clinically obvious manifestations over healthy men whose test indicators did not exceed the physiological norm was established. Based on this, it was interesting to look at other independent emotionally negative risk factors for CHD of people under our supervision (such as reduced stress tolerance and increased anxiety) through the prism of alexithymia, for which all respondents were divided into three subgroups, depending on the presence and severity of alexithymia in them. This approach proved to be justified since it allowed us to identify the following trends: as the severity of alexithymia increased, there was an increase in the level of anxiety as a disposition and a decrease in the level of resistance to the action of stress agents in the individuals we examined. The abovementioned increases the relevance of the expediency of the psychological support for clinically healthy men who either have a tendency to develop alexithymia or its obvious clinical signs.

\section{CONCLUSION}

Thus, as the severity of alexithymia increased, there was an increase in the level of anxiety as a disposition and a decrease in the level of resistance to the action of stress agents in the healthy male respondents 
we observed which may increase the risk of developing CHD in them. Therefore, clinically healthy individuals who have a tendency to develop alexithymia or its obvious manifestations need psychological correction of the personal changes established in them to increase the effectiveness of primary prevention of CHD.

\section{REFERENCES}

1. Albus C., Herrmann-Lingen C., Jensen K. et AL. Additional effects of psychological interventions on subjective and objective outcomes compared with exercise-based cardiac rehabilitation alone in patients with cardiovascular disease: A systematic review and meta-analysis. Eur J Prev Cardiol 2019. Doi: $10.1177 / 2047487319832393$.

2. Martinez-Sanchez F. The Spanish version of the Toronto Alexithymia Scale (TAS-20). Clinica y Salud 1996; 7: 19-32.

3. Bezverkhov A. A., IshChenko O. Yu. Dynamics of mortality from cardiovascular diseases for the period 2015-2019. Norwegian Journal of Development of the International Science 2021; 54: 35-38. Doi: 10.24412/3453-9875-2021-54-2-35-38.

4. Velikanov A. A., Stolyarova A. A., Kruglova N. E. AND OTHERS. Features of the psychoemotional sphere of patients with coronary heart disease: a review of studies. Psychology. Psychophysiology. 2020; 13(1): 23-33. Doi: 10.14529/jpps200103.

5. Garganeeva N. P., Kornetov N. A., BelokryLOVA M. F. Psycho-social factors, anxiety and depressive disorders of patients with coronary heart disease: problems of comorbidity and prognosis. Russian Journal of Cardiology 2020; 25 (9): 26-32. Doi: 10.15829/1560-4071-2020-404.
6. Kayumova M. M., Gakova E. I., Senatorova O. V. Epidemiological aspects of the prevalence of coronary heart disease in an open urban population: gender differences. Siberian Medical Journal 2019; 34(2): 146-151. Doi: 10.29001/2073-8552-2019-342-146-151.

7. Kodochigova A. I., Kirichuk V. F., Sinkeev M. S., ETC. Features of the psychological status of patients with various forms of coronary heart disease. Saratov Scientific and Medical Journal 2019; 15 (3): 744-749.

8. Kodochigova A. I., Kirichuk V. F., Dzheyranova M. O. AND OThers. Psychosocial risk factors for recurrent acute coronary catastrophe in patients with coronary heart disease: a view through the prism of alexithymia. Therapy 2021; 3: 43-52. Doi: $10.18565 /$ therapy. 2021. 3. 43-52.

9. Severova E. A., Okhapkin A. S., Dautova M. A. AND OTHERS. Alexithymia as a phenomenon that combines some aspects of mental and psychosomatic pathologies. Bulletin of the Smolensk State Medical Academy 2016; 15(4): 126-133.

10. Subbotin S. V. Resistance to mental stress as a characteristic of a teacher's meta-identity: Dis. cand. psychol. Science, Perm, 1993; 152.

11. Khanin Yu. L. A brief guide to the use of the scale of reactive and personal anxiety by $\mathrm{Ch}$. D. Spielberger. L.: LNII FC 1976; 18.

There is no conflict of interest in the article. 